\title{
Neuroscience Domain
}

National Cancer Institute

\section{Source}

National Cancer Institute. Neuroscience Domain. NCI Thesaurus. Code C95096.

A subject domain utilized for the submission of information encompassing and

representing data, vocabulary or records related to the brain and nervous system. 